\title{
Tagung Dermatologische Praxis 2017
}

\author{
Bei der Dermatologischen Praxis in Frankenthal konnten die Teilnehmer wieder aus \\ einer Vielzahl an unterschiedlichen Veranstaltungen mit über 70 Referenten auswählen. \\ Ein Schwerpunkt war am Samstag die Behandlung der Akne. Ebenfalls am Samstag \\ hatten die Besucher auch Gelegenheit, sich in einem Workshop der Arbeitsgemeinschaft \\ Ästhetische Dermatologie und Kosmetologie (ADK) aus erster Hand über wichtige Themen \\ der ästhetischen Medizin zu informieren.
}

\section{Europäische Leitlinie zur Therapie der Akne: Was ist neu?}

Die Verbesserung der Behandlung, die Verminderung von schweren Verläufen und von Narbenbildungen, die Unterstützung der Adhärenz und das Verhindern von Antibiotika-Resistenzen sind Ziele der aktualisierten europäischen S3-Leitlinie des European Dermatology Forum (EDF) zur Therapie der Akne $[1,2]$. Für das Update wurden ausschließlich randomisierte kontrollierte klinische Studien (RCT) sowie zwei CochraneReviews berücksichtigt und dabei erstmals auch die verfügbare Literatur zur Erhaltungstherapie ausgewertet, berichtete Prof. Falk Ochsendorf, Universitäts-Hautklinik Frankfurt. Zu den wesentlichen allgemeinen Aussagen zählen:

- Systemische Monotherapien sind nicht effektiver als topische Therapeutika.

- Die Kombination topischer und systemischer Ansätze ist einer systemischen Monotherapie überlegen.

- Fixkombinationen sind besser als topische Monotherapien mit den Komponenten.

Mit einem hohen Empfehlungsgrad werden für die Behandlung der milden bis moderaten Acne papulopustulosa die Fixkombinationen Adapalen plus Benzoylperoxid (BPO) sowie BPO plus Clindamycin genannt. Die moderate noduläre Akne sowie alle schweren Formen sollten frühzeitig systemisch mit Isotretinoin behandelt werden. Mit einem mittleren Empfehlungsgrad werden bei der Acne comedonica topische Retinoide, bei der milden bis moderaten Acne papulopustulosa alternativ Azelainsäure, BPO, topische Retinoide, eine Fixkombination aus topischem Clindamycin und Tretinoin oder - vor allem wenn die Akne flächenhaft zum Beispiel am Rücken auftritt ein systemisches Antibiotikum plus Adapalen angeraten. Bei schwerer Acne papulopustulosa und moderater nodulärer Akne wird mit einem mittleren Empfehlungsgrad die Kombination eines systemischen Antibiotikums mit Adapalen, mit Azelainsäure oder mit einer Adapalen-BPO-Fixkombination genannt. Ein systemisches Antibiotikum plus Azelainsäure oder Adapalen-BPO-Fixkombination sind auch Optionen mit mittlerem Empfehlungsgrad bei der schweren nodulären Akne und der Acne conglobata. Unter den topischen Retinoiden sollte Adapalen bevorzugt werden, da es besser verträglich ist, erläuterte Ochsendorf. Antibiotikum der Wahl ist Doxycyclin; es sollte jedoch nicht länger als drei Monate gegeben werden, da dann kein zusätzlicher Nutzen zu erwarten sei und die Gefahr von Resistenzen steigt.

Nicht empfohlen werden topische oder systemische Antibiotika als Monotherapie, künstliche UV-Bestrahlung sowie Monotherapien mit hormonellen Antiandrogenen.

Als Erhaltungstherapie werden bei der Acne comedonica Azelainsäure und topische Retinoide empfohlen, bei der milden bis moderaten Acne papulopustulosa neben diesen beiden Arzneimitteln auch BPO. Bei moderater nodulärer Akne sowie allen schweren Formen können eine Adapalen-BPO-Fixkombination, Azelainsäure, BPO, niedrig dosiertes systemisches Isotretinoin oder ein topisches Retinoid sowie bei Frauen Antiandrogene in Kombination mit Topika eingesetzt werden. Die Studienlage bezüglich der Erhaltungstherapie reiche bislang allerdings weder für hoch- noch mittelgradige Empfehlungen aus, berichtete Ochsendorf.

Angelika Bauer-Delto

\footnotetext{
1. http://www.euroderm.org/edf/index.php/edf-guidelines/category/4-guidelines-acne

2. Nast A et al.: JEADV 2016; 30: 1261-1268 (Short Version)
}

Vortrag „Update Akne S3-Leitlinie: Was ist neu?" von Prof. Falk Ochsendorf, 18. März 2017

\section{Die Adhärenz junger Akne-Patienten verbessern - aber wie?}

In der Praxis sei ein therapeutischer Nihilismus nicht angebracht und auch kein vertrösten, dass die Zeit die Akne schon heilen wird, betonte Dr. Roland Aschoff, Universitäts-Hautklinik Dresden. Die Lebensqualität wird durch eine Akne erheblich beeinträchtigt und rund ein Drittel der Patienten leidet unter psychischen Komorbiditäten wie Angststörungen, Depressionen und suizidalen Tendenzen. Eine erfolgreiche Behandlung verkürzt den Verlauf, mildert die Schwere der Erkrankung und kann Narben verhindern - und nicht zuletzt bessert sie das Selbstbewusstsein und die soziale Kontaktfähigkeit der meist jungen Patienten.

Ein großes Problem in der Praxis sei jedoch die oft geringe Adhärenz. Nur die Hälfe aller Jugendlichen wendet die Therapie wie empfohlen an. Gründe können sein, dass die meist jungen Patienten oft wenig Kenntnisse über den Krankheitsverlauf und die Therapie haben oder auch Schwierigkeiten, die Behand- 